\title{
Paralelização Assíncrona da Programação Dinâmica Dual com Agrupamento Ótimo de Nós
}

\author{
Lucas R. Conceição* André L. Diniz** \\ André L. M. Marcato ${ }^{* * *}$ Amanda Pavila ${ }^{* * * *}$ \\ * Faculdade de Engenharia Elétrica, Universidade Federal de Juiz de \\ Fora, MG, (e-mail: lucasrcon@gmail.com). \\ ** Centro de Pesquisas de Energia Elétrica, DEA - Departamento de \\ Otimização Energética e Meio Ambiente, RJ(e-mail: diniz@cepel.br) \\ *** Faculdade de Engenharia Elétrica, Universidade Federal de Juiz de \\ Fora, MG, (e-mail: andre.marcato@ufjf.edu.br ) \\ **** Faculdade de Engenharia Elétrica, Universidade Federal de Juiz de \\ Fora, MG, (e-mail: amanda.pavila@engenharia.ufjf.br)
}

\begin{abstract}
The hydrothermal coordination problem of real systems has a great complexity. This complexity is linked to the need of model the hydrological uncertainties of the systems. For this purpose, a tree of possible inflow scenarios is built. In order to solve this problem several methodologies can be applied, including Dual Dynamic Programming (PDD). Although, for large problems, such as the Brazilian system, this methodology results in an elevated time of resolution. The work presents a methodology with the objective of reduce this time through the asynchronous parallelization of the PDD, together with the optimum grouping of the nodes of the scenario tree in subproblems by using a genetic algorithm.

Resumo: O problema de planejamento energético de sistemas hidrotérmicos reais possui uma grande complexidade, a qual está ligada à necessidade de se modelar as incertezas hidrológicas. Logo, é realizada a construção de uma árvore de possíveis cenários de afluências às usinas hidrelétricas. Para a solução deste problema várias metodologias podem ser aplicadas, entre elas a Programação Dinâmica Dual (PDD), porém para problemas grandes, como o do sistema brasileiro, esta metodologia pode demandar um esforço computacional elevado. O trabalho apresenta uma metodologia com o objetivo de diminuir o tempo de processamento através da paralelização de forma assíncrona da PDD juntamente com o agrupamento ótimo dos nós da árvore de cenários em subproblemas, por meio de algoritmo genético.
\end{abstract}

Keywords: Hydrothermal Systems Planning, Dual Dynamic Programming, Asynchronous Parallelization, Genetic Algorithm.

Palavras-chaves: Planejamento Sistemas Hidrotérmicos, Programação Dinâmica Dual, Paralelização assíncrona, Algoritmo Genético.

\section{INTRODUÇÃO}

O território brasileiro possui características particulares que fazem com que seu sistema elétrico seja diferenciado em relação aos demais países do mundo. De acordo com [ONS, 2019] o sistema de produção e transmissão de energia elétrica do Brasil é um sistema hidro-termo-eólico de grande porte, com predominância de usinas hidrelétricas e com múltiplos proprietários. O Sistema Interligado Nacional (SIN) é constituído por quatro subsistemas: Sul, Sudeste/Centro-Oeste, Nordeste e a maior parte da região

\footnotetext{
* Este trabalho teve o suporte financeiro da Coordenação de Aperfeiçoamento de Pessoal de Nível Superior (CAPES), do Conselho Nacional de Desenvolvimento Científico e Tecnológico (CNPq), do Centro de Pesquisas de Energia Elétrica (CEPEL), da Fundação de Amparo à Pesquisa do Estado de Minas Gerais (FAPEMIG), do Instituto Nacional de Energia Elétrica (INERGE) e da Agência Nacional de Energia Elétrica (ANEEL).
}

Norte. Ainda segundo [ONS, 2019] a capacidade instalada de geração do SIN é composta, principalmente, por usinas hidrelétricas distribuídas em dezesseis bacias hidrográficas nas diferentes regiões do país. As usinas térmicas, em geral localizadas nas proximidades dos principais centros de carga, desempenham papel estratégico relevante, pois contribuem para a segurança do SIN. Essas usinas são despachadas em função das condições hidrológicas vigentes, permitindo a gestão dos estoques de água armazenada nos reservatórios das usinas hidrelétricas, para assegurar o atendimento futuro.

Realizar o planejamento da operação do SIN está ligado, portanto, à questão de quanto e quando produzir a energia necessária ao atendimento da demanda pelo menor custo total de operação possível. O quanto produzir está associado à quantidade de energia a ser gerada pelas hidrelétricas e também pelas termelétricas. A determinação de quando 
produzir refere-se à decisão de qual o melhor momento de se gerar pelas usinas hidrelétricas, dada a ocorrência de chuva em cada período de tempo analisado. Devido as características particulares já descritas do SIN, a coordenação da operação e o planejamento do mesmo tornam-se problemas complexos, o que levou à necessidade de construção de modelos específicos para gerenciar a operação elétrica e energética do sistema. O problema é resolvido em diferentes modelos de acordo com o horizonte de estudo, sendo eles de longo, médio e curto prazos. Todos estes modelos computacionais são operados de forma coordenada visando minimizar os custos globais do sistema como um todo, de acordo com [MARCATO, 2002] e [MACEIRA, 2002], a interconexão entre os sistemas elétricos e a integração dos recursos de geração e transmissão são de suma importância para o planejamento e redução de custos, pois em períodos hidrológicos desfavoráveis em determinadas regiões, outras podem estar em situação hidrológica favorável, reduzindo a necessidade da utilização de energia térmica. Por outro lado, em períodos de déficit hidrológicos as térmicas contribuem para o atendimento da carga.

A representação do sistema, portanto, varia de acordo com o horizonte a ser analisado. Nos modelos de longo e médio prazo as usinas hidrelétricas são representadas de forma simplificada através de sistemas equivalentes de energia enquanto a estocasticidade das afluências é representada de forma mais detalhada. No modelo de curto prazo, a incerteza com relação as afluências futuras tendem a diminuir já que o horizonte de estudo considerado diminui, porém, por este mesmo motivo, as representações das usinas do sistema passam a ter a necessidade de serem mais detalhadas. Para representar a estocasticidade do problema, uma árvore de cenários é montada, nesta árvore cada nó possui um possível cenário de vazões afluentes com o objetivo de modelar as incertezas hidrológicas inerentes ao problema.

Quando se considera o problema de planejamento energético de sistemas hidrotérmicos de sistemas reais tem-se um número muito grande de estágios e possíveis cenários de afluências, o que resulta em uma árvore de cenários com muitos nós. Por conta disso, a formulação equivalente determinística deste problema é alcançada por um Problema Linear (PL) de grandes dimensões, uma vez que o mesmo incluiria as restrições e contribuições para a função objetivo relacionada à todos os cenários, com suas probabilidades correspondentes, da árvore. Assim, dependendo do problema, o tamanho deste PL pode se tornar proibitivo. Para solucionar esta questão, algoritmos de decomposição têm sido propostos na literatura como uma alternativa para resolver esses grandes problemas nos quais a abordagem equivalente e determinística provou ser ineficiente ou intratável.

Dentro dos vários algoritmos de decomposição que podem ser utilizados para solucionar o problema destacase a decomposição por cortes de Benders [BENDERS, 1962]. Técnica na qual o conjunto de variáveis de decisão é explicitamente dividido de acordo com os estágios do problema e as chamadas variáveis de estado conectam estes estágios, que são resolvidos iterativamente até a convergência do problema. A decomposição de Benders para vários estágios, também conhecida como Programação Dinâmica Dual (PDD) [BIRGE, 1985], é amplamente utilizada para resolução de problemas lineares ou convexos com uma representação em árvore de cenários. Na forma tradicional desta abordagem, o problema geral é decomposto de acordo com os nós da árvore de cenários. Cada estágio da árvore corresponde às decisões em um determinado período de tempo e os ramos representam os possíveis cenários para cada estágio. Considerando isso, cada nó é resolvido separadamente enquanto o algoritmo evolui ao longo da árvore e as informações locais são transmitidas em processos forwards e backwards até que uma solução ótima global seja encontrada dentro de uma tolerância desejada.

No entanto, a resolução de problemas estocásticos através da PDD pode exigir um número considerável de iterações até que a convergência seja alcançada. Como é desejável resolver os problemas de grande proporção, sem se comprometer o tempo necessário para se obter a convergência do mesmo, várias técnicas para melhorar a eficiência dos algoritmos originais foram propostos na literatura com o objetivo de reduzir esse tempo necessário para a solução do problema [BRANDÃO, 2018], seja por meio da redução do número de iterações, do tempo de cada iteração, do número de operações necessárias, entre outras estratégias.

Dentro das estratégias para acelerar o tempo de solução do problema, este trabalho dará destaque à paralelização da metodologia PDD, abordagem já considerada em trabalhos como [DEMPSTER, 1999], [JESÚS, 2008], [SANTOS, 2017] e [BRANDÃO, 2018]. A paralelização leva à uma melhoria no tempo de resolução em problemas que compreendem tarefas independentes, e sua implementação só é possível através da utilização de computadores que compreendem muitos processadores, que operam simultaneamente, ou de clusters computacionais. O trabalho propõe a paralelização assíncrona da $\mathrm{PDD}$, como proposto em [SANTOS, 2017] e [BRANDÃO, 2018], com o objetivo de quebrar a dependência temporal do algoritmo PDD tradicional e, assim, aumentar sua adequação para ambientes paralelos.

A paralelização aplicada à PDD requer sincronização entre o processador mestre e os demais processadores alocados para os subproblemas, o que pode tornar a implementação para problemas em múltiplos estágios, inviável. Para solucionar este problema, técnicas de agregação de nós podem ser aplicadas, como abordado em [JESÚS, 2008] existem diversas técnicas de agrupamentos diferentes, como o agrupamento por decomposição por nó, decomposição por cenário, por sub-árvore, e por cenário completo. O trabalho propõe utilizar um algoritmo genético para definir o agrupamento ótimo para o problema de acordo com o número de estágios a serem estudados.

O principal objetivo deste trabalho portanto, é o de propor novas técnicas capazes de melhorarem a eficiência do método PDD. Para isso, o trabalho faz a implementação da paralelização deste método de forma assíncrona juntamente com a agregação de nós de forma ótimizada através da utilização de um algoritmo genético responsável por encontrar o agrupamento de nós ótimo para a árvore de cenários para o problema de acordo com o número de estágios a serem estudados.

O trabalho concentra-se no modelo de otimização para o horizonte de curto prazo, cujo objetivo é determinar 


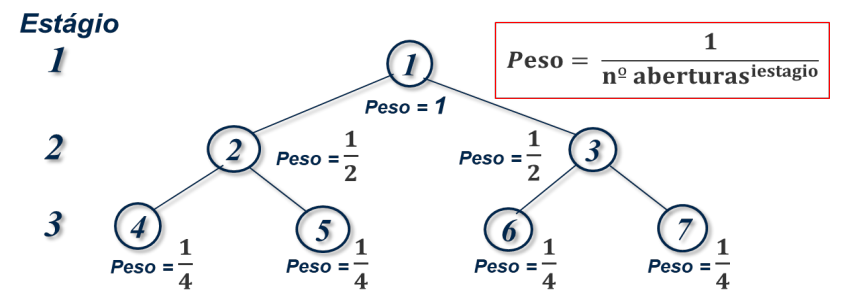

Fig. 1. Pesos para árvore de cenários

o despacho de geração das usinas hidráulicas e térmicas que minimiza o custo de operação ao longo do período de planejamento. As usinas hidrelétricas e termelétricas são representadas como parques geradores individualizados e a aleatoriedade das vazões é representada através de uma árvore de possíveis cenários de vazões afluentes, além disso, são consideradas restrições para atender o balanço hídrico e a demanda do sistema, assim como as restrições relacionadas às características físicas das usinas, como por exemplo, limites de geração.

O item 2 irá descrever todas as metodologias utilizadas neste trabalho para solucionar o problema de árvore completa. Também será apresentado o algoritmo genético implementado para otimizar o agrupamento de nós da árvore de cenários.

\section{METODOLOGIAS}

O problema foi resolvido de três maneiras diferentes. Primeiramente modelou-se o problema como um problema linear único no qual cada variável de decisão possui um peso correspondente à sua probabilidade de ocorrência. Depois, resolveu-se o mesmo problema através da Programação Dinâmica Dual (PDD), implementada através de uma função recursiva que está detalhada no item $2.2 \mathrm{e}$, por fim, realizou-se a paralelização de forma assíncrona da PDD, com o objetivo de diminuir o tempo gasto para a convergência do problema.

A seguir as metodologias implementadas serão detalhadas com base em uma árvore de cenários simples, com o intuito de tornar mais didática a explicação do funcionamento dos algoritmos implementados.

\subsection{Programação Linear}

Um Problema de Programação Linear (PL) é o nome dado à problemas de otimização que consistem em encontrar o melhor valor possível para uma função objetivo linear de " $n$ " variáveis respeitando uma série de restrições. Para resolver o problema de árvore completa como um único PL, todos os estágios são somados e suas restrições de balanço hídrico, atendimento a demanda e de canalização são adotadas, considerando pesos referentes às probabilidades de ocorrência de cada possível cenário da árvore. Desta forma, para uma árvore simples com três estágios e duas aberturas, com nós equiprováveis, como mostrado na figura 1, a formulação completa para o sistema teste é o apresentado nas equações 1 e 2, função objetivo e restrições respectivamente.

$$
\begin{aligned}
& \operatorname{minf}= c_{g t 1} \times g t_{11}+c_{g t 2} \times g t_{21}+c_{d e f} \times d e f_{1}+ \\
& \frac{1}{2}\left[\left(c_{g t 1} \times g t_{12}^{1}+c_{g t 2} \times g t_{22}^{1}+c_{d e f} \times d e f_{2}^{1}\right)+\right.\left(c_{g t 1} \times g t_{12}^{2}+c_{g t 2} \times g t_{22}^{2}+c_{d e f} \times d e f_{2}^{2}\right)+ \\
& \frac{1}{4}\left[\left(c_{g t 1} \times g t_{13}^{1}+c_{g t 2} \times g t_{23}^{1}+c_{d e f} \times d e f_{3}^{1}\right)+\right. \\
&\left(c_{g t 1} \times g t_{13}^{2}+c_{g t 2} \times g t_{23}^{2}+c_{d e f} \times d e f_{3}^{2}\right)+ \\
&\left(c_{g t 1} \times g t_{13}^{3}+c_{g t 2} \times g t_{23}^{3}+c_{d e f} \times d e f_{3}^{3}\right)+ \\
&\left(c_{g t 1} \times g t_{13}^{4}+c_{g t 2} \times g t_{23}^{4}+c_{d e f} \times d e f_{3}^{4}\right)
\end{aligned}
$$

sujeito a:

$$
\begin{gathered}
g t_{1 j}^{i}+g t_{2 j}^{i}+\rho \times v_{t j}^{i}+d e f_{j}^{i}=D_{j}^{i} \\
v_{f j}^{i}+v_{t j}^{i}+v_{v j}^{i}-V I_{j}^{i}=A F L_{j}^{i} \\
x_{\text {minj }}^{i} \leq x_{j}^{i} \leq x_{\operatorname{maxj}}^{i}
\end{gathered}
$$

onde:

$\mathrm{i}=$ número do estágio

$\mathrm{j}=$ número da abertura

$\mathrm{gt}_{1 j}^{i}=$ variáveis de decisão da geração da térmica 1

$g t_{2 j}^{i}=$ variáveis de decisão da geração da térmica 2

$v_{t j}^{i}=$ variáveis de decisão do volume turbinado da hidrelétrica

$v_{f j}^{i}=$ variáveis de decisão do volume final da hidrelétrica

$v_{v j}^{i}=$ variáveis de decisão do volume vertido da hidrelétrica

$V I_{j}^{i}=$ valor do volume inicial da hidrelétrica

$\rho \stackrel{j}{=}$ valor da produtibilidade da hidrelétrica

$A f l_{j}^{i}=$ valor da afluência à usina hidrelétrica

$\operatorname{de} f_{j}^{i}=$ variáveis de decisão do déficit

$D_{j}^{i}=$ valor da demanda do sistema

A partir das equações 2 e 3, evidencia-se que devido a dimensão do problema, este tipo de solução se torna inviável para problemas de planejamento de sistemas hidrotérmicos reais. Esta inviabilidade, só reforça a necessidade da utilização de metodologias de decomposição, tais como a PDD, para solucionar problemas mais complexos, com mais estágios e mais aberturas.

\subsection{Programação Dinâmica Dual}

A Programação Dinâmica Dual (PDD) faz uso da Decomposição de Benders, que consiste em dividir o problema em diversos estágios, no qual cada estágio é dependente do estágio subsequente, não sendo necessário a discretização de todo o espaço de estado do sistema em questão. Com isso, informações obtidas no período corrente são repassadas para períodos posteriores permitindo que haja influências e interferências entre estes períodos. Isto é, permitindo que o futuro tenha informações do passado. Desta forma, na Programação Dinâmica Dual são realizados dois processos: forward e backward.

O processo forward é iniciado no primeiro estágio e finalizado no último estágio. Neste processo ocorre uma recursão direta, não incluindo informações a respeito do custo futuro da operação, sendo o mesmo resolvido através de técnicas de programação linear. Desta forma, a tendência do modelo é a utilização, em cada estágio, da capacidade máxima das usinas hidrelétricas, o que consequentemente levará à utilização de usinas termelétricas nos últimos estágios e a um elevado custo futuro de operação, ou até mesmo um possível déficit.

No processo backward segue-se caminho contrário ao anterior, iniciando no último estágio e finalizando no primeiro. A cada novo estágio é gerada uma restrição com relação ao estágio anterior, contruída através de cortes de Benders. Esta restrição trará informações importantes sobre o custo futuro e consequentemente à otimização da operação do sistema hidrotérmico. A figura 2 apresenta o fluxograma da PDD. 


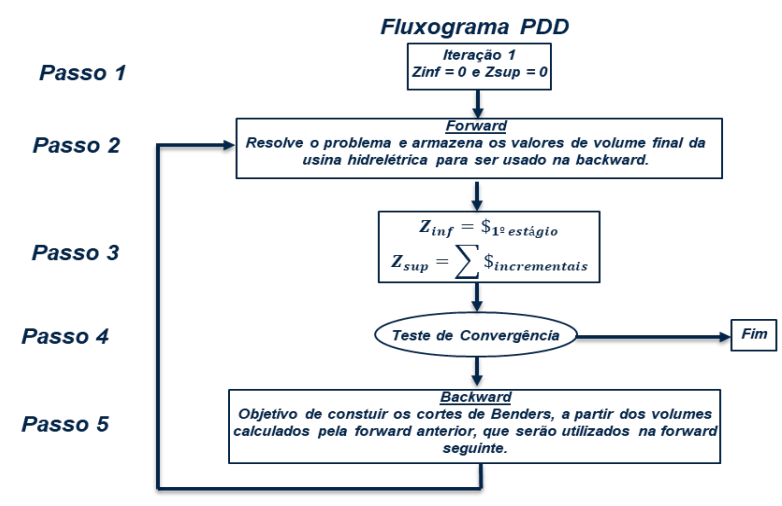

Fig. 2. Fluxograma PDD

O objetivo do problema, como visto anteriormente, é minimizar o valor esperado do custo de operação nos meses de estudo. Para isso, no Passo 3, calcula-se o valor do limite inferior (Zinf) considerando que ele corresponde ao custo esperado total do primeiro estágio e o valor do limite superior (Zsup), que é calculado como o somatório dos custos imediatos de cada estágio. Para o caso da árvore completa, este cálculo é feito através do somatório dos valores médios de cada cenário de afluência.

No Passo 4 é verificado se a diferença entre eles é menor que uma tolerância pré-determinada. Se isso ocorre, o conjunto de soluções obtido é a solução ótima, e considera-se que ocorreu a convergência do algoritmo. Caso contrário, inicia-se o processo backward, o qual criará um corte de Benders para cada estágio recursivo anterior. O Passo 5 corresponde ao processo backward, no qual são formadas as restrições para os próximos estágios, com base nas soluções ótimas obtidas pelo processo forward (Passo 2). Como este problema corresponde a um processo iterativo, a função que possui a informação do custo futuro vai sendo aproximada pelos cortes de Benders. O próximo passo é uma nova iteração, que retorna ao processo forward, retorna ao Passo 2, no qual inclui os cortes criados como restrições, para o primeiro estágio até o último. Uma nova solução ótima é encontrada para as variáveis de decisão e este processo prossegue, a cada iteração, até que a diferença entre o limite inferior (Zinf) e o limite superior (Zsup) seja igual à tolerância adotada, ponto este que corresponde à convergência do processo.

Neste trabalho implementou-se o algoritmo PDD através de uma função recursiva, tornando a implementação mais simples e robusta. A figura 3 mostra o funcionamento do algoritmo implementado, notase que a solução das etapas forward e backward se misturam durante a solução do problema.

No $1^{\circ}$ passo, o problema para o primeiro estágio é resolvido, dando início ao processo forward, e o valor encontrado para o volume final da usina hidrelétrica é passado para os nós do estágio seguinte para serem utilizados como volumes iniciais. A partir dele a árvore é percorrida até o último estágio, como pode ser visto nos $2^{\circ}$ e $3^{\circ}$ passos, ao chegar em um problema de fim de árvore o processo backward começa a ocorrer através da construção dos cortes de Benders. No $4^{\circ}$ passo vemos que o algoritmo percorre todas as aberturas do ramo da árvore em que se encontra, para ao fim de percorre-los calcular o corte médio que será utilizado pelo nó do estágio anterior. Com este corte médio, este nó resolve o problema mais uma vez, agora com a nova restrição do corte médio recebido por ele. Ao final da solução deste novo problema, o corte referente ao estágio atual é calculado, como visto no passo 5 . Nos $6^{\circ}, 7^{\circ}, 8^{\circ}$ e $9^{\circ}$ passos, o processo descrito é repetido para o segundo ramo da árvore definido a partir da segunda abertura do segundo estágio. Por fim, no $10^{\circ}$ passo, o problema backward para o primeiro estágio é resolvido a partir do corte médio construído durante todo o processo forward e backward dos demais estágios.

O teste de convergência é realizado com o valor de Zinf e Zsup médios calculados durante a iteração, o valor de Zsup médio é encontrado

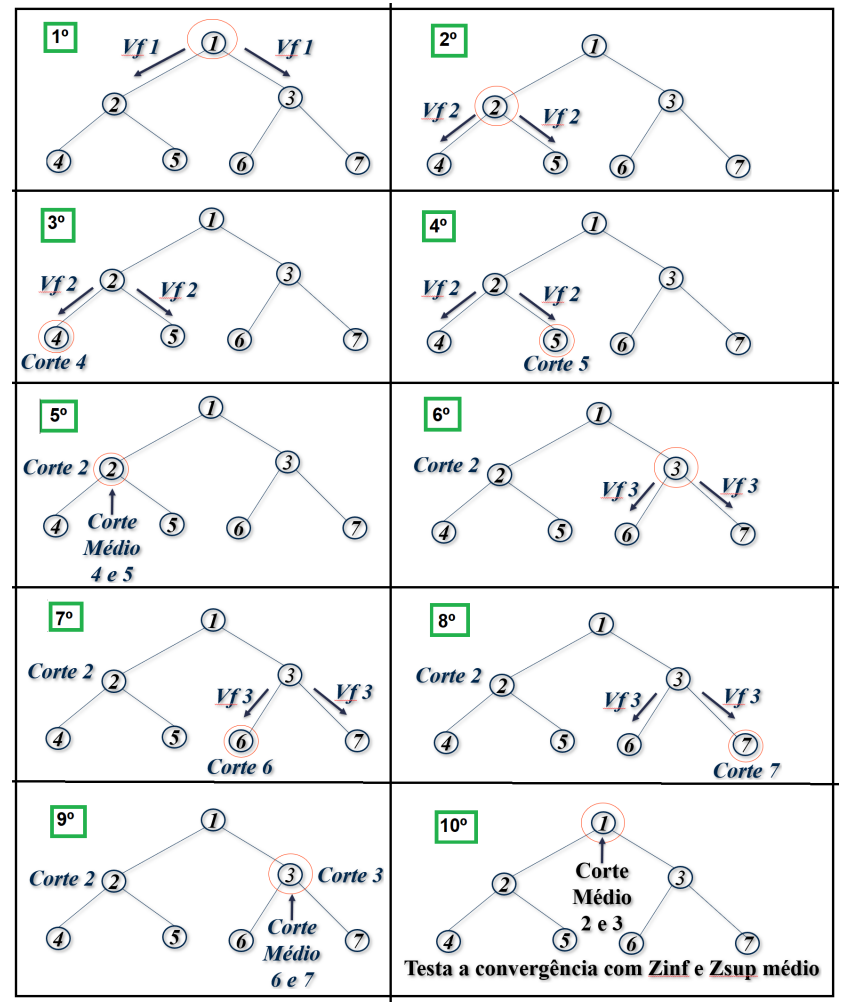

Fig. 3. Resolução da PDD com função recursiva

realizando a média entre os valores dos custos imediatos encontrados em cada nó da árvore. Caso não haja convergência todo o processo é repetido considerando as restrições construídas durante a primeira iteração.

Para problemas mais complexos, nos quais o número de estágios e aberturas são maiores, o tempo computacional necessário para encontrar a solução pela PDD aumenta consideravelmente. Com o intuito de solucionar este problema, realizou-se a paralelização assíncrona da metodologia, a qual será apresentada a seguir.

\subsection{PDD paralela assíncrona}

Um modo clássico de paralelizar a PDD foi proposto por [DEMPSTER, 1998], ele explora a independência dos cenários no mesmo estágio da árvore. A ideia principal é aplicar o algoritmo PDD tradicional, resolvendo os nós no mesmo estágio em paralelo. Como o subproblema de um nó requer, durante o passo forward da solução, o estado de seu nó ascendente ou, durante o passo backward da solução os cortes de Benders de seus nós descendentes, existe uma dependência entre estágios que cria um ponto de sincronização em cada estágio, esses pontos de sincronização são uma grande desvantagem deste método [BRANDÃO, 2018]. Além de criar uma dependência entre os estágios durante o processo backward e o processo forward, o método clássico também limita a granularidade do algoritmo, uma vez que a granularidade máxima de um estágio será dada pelo número de cenários nesse nível. Portanto, como cada estágio tem uma granularidade diferente, espera-se a ociosidade ao longo do procedimento tradicional de solução da PDD, especialmente nos primeiros cenários da árvore de cenários.

Por esse motivo a abordagem escolhida para este trabalho foi a de paralelizar a PDD de forma assíncrona, como proposto em [SANTOS, 2017] e [BRANDÃO, 2018]. O algoritmo da PDD paralelizado de forma assíncrona não possui um passo forward e backward, e por conta disso sua iteração ocorre de forma diferente da iteração do modelo tradicional. Suas iterações são definidas por etapas, caracterizadas pela resolução independente de todos os subproblemas da árvore de cenário. A troca de informações ocorre simultaneamente 


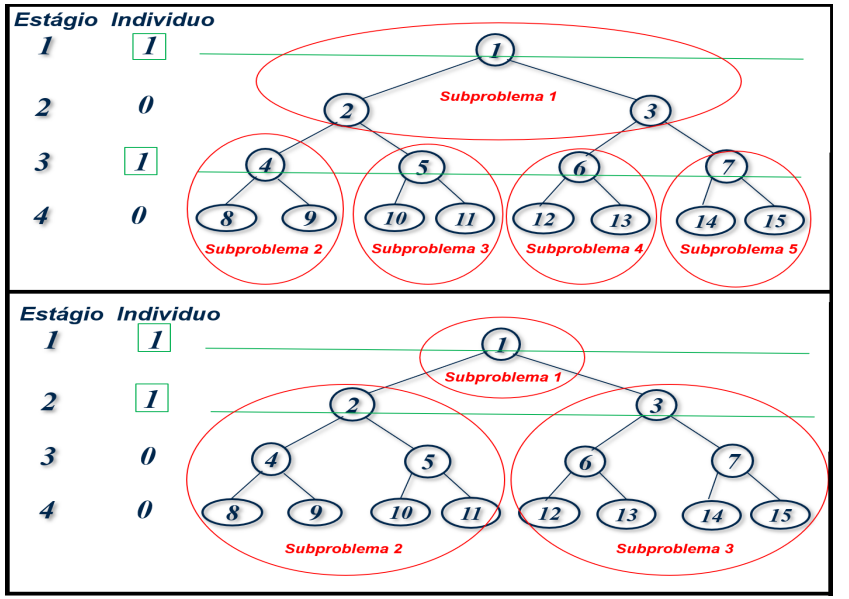

Fig. 4. Exemplos de divisão de subproblemas

ao longo da árvore entre as iterações ou etapas, as variáveis de estado com a informação dos volumes finais passam para os estágios descendentes e os cortes de Benders para os estágios ascendentes de cada subproblema. Desta forma os nós são totalmente independentes entre si e podem ser resolvidos simultaneamente dentro de um passo que definirá a resolução de uma iteração, porém entre os passos há um ponto de sincronização.

Desta maneira, implementou-se a paralelização assíncrona a partir da implementação recursiva da PDD apresentada na seção anterior. Para realizar a distribuição dos subproblemas entre os processadores definiu-se uma variável auxiliar (individuo). Esta variável defini em quais estágios da árvore ocorrerá corte para o agrupamento dos nós em sub-árvores para serem resovidas como subproblemas. Na figura 4 temos um exemplo deste agrupamento para um problema de quatro estágios e duas aberturas, a variável individuo determina as posições de início de sub-árvore adotando a informação 1 para tal, desta forma o primeiro estágio sempre será igual a 1 enquanto os demais estágios dependerão da escolha do usuário.

No primeiro exemplo da figura 4 , o corte foi realizado no primeiro e terceiro estágios, criando assim um subproblema entre o primeiro e segundo estágios e quatro subproblemas entre o terceiro e quarto estágios. Já no segundo exemplo o corte foi realizado no primeiro e segundo estágios, criando assim um subproblema entre primeiro e segundo estágios e dois subproblemas entre o segundo e quarto estágios.

Cada subproblema será enviado a processadores diferentes para serem resolvidos simultaneamente, além disso, o processador mestre ficará por conta de realizar as sincronizações ao final de cada iteração. A figura 5 ilustra o funcionamento do algoritmo PDD funcionando de forma paralelizada.

O funcionamento é similar ao do algoritmo PDD com função recursiva mostrado na seção anterior. Para inicializar os valores dos volumes iniciais realiza-se um passo forward tradicional e com os valores de volumes iniciais calculados o processo de solução é iniciado. No $1^{\circ}$ passo os nós $1,4,5,6$ e 7 serão resolvidos de forma simultânea. Os resultados encontrados para a variável de estado que representa o volume final serão passados para seus estágios descendentes pertencentes a seu subproblema, como mostrado no primeiro exemplo da figura 4 . Nos $2^{\circ}, 3^{\circ}$ e $4^{\circ}$ passos ocorre a resolução dos problemas dos nós descendentes, que no exemplo da figura 5 , são todos nós de fim de árvore para seus subproblemas. Ao final do $4^{\circ}$ passo os cortes médios de Benders já são calculados e passados para os nós ascendentes para que os mesmos possam resolver o problema mais uma vez considerando o corte médio recebido, $5^{\circ}$ passo. Se os nós ascendentes não forem o primeiro estágio, eles irão calcular seus cortes para ao final da iteração construir os cortes médios a serem utilizados pelos seus nós ascendentes nas próximas iterações, neste exemplo isto ocorre para os nós $4,5,6$ e 7 .

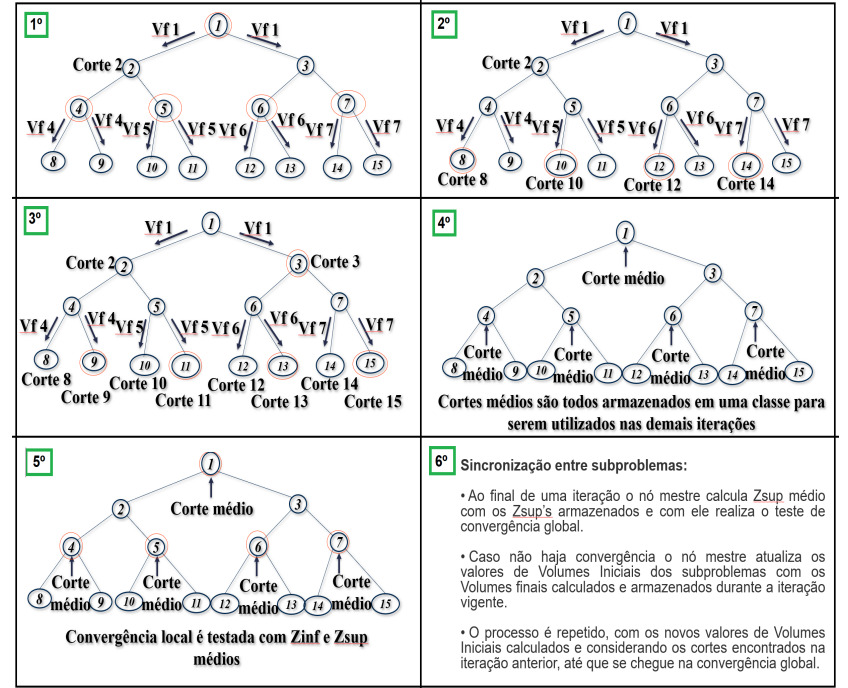

Fig. 5. Funcionamento PDD paralela assíncrona

Ao final do $5^{\circ}$ passo um teste de convergência local é realizado comparando-se os valores dos limites inferiores (Zinf's) e os valores dos limites superiores médios (Zsup's), valores esses calculados em cada subproblema. Os subproblemas só retornam as informações de cortes médios, Zsup's médios e volumes finais (Vf's) calculados para o processador mestre realizar a etapa de sincronização, quando suas convergências locais ocorrem. Por fim, no $6^{\circ}$ passo ocorre a sincronização dos resultados, primeiramente o processador mestre verifica se existe convergência global através dos valores do limite inferior global (Zinf do estágio 1) e do limite superior médio (Zsup médio, calculado com os dados coletados dos demais processadores). Caso não tenha ocorrido convergência, ele distribui os dados coletados para os demais processadores e uma nova iteração do problema é inicializada. Desta vez, considerando os cortes calculados na iteração anterior e os novos valores de volume inicial também retirados das informações da primeira iteração. O processo é repetido até que ocorra convergência global.

Para problemas com árvores de cenários mais complexas, maior número de estágios e aberturas, o número de maneiras de se agrupar os nós em subproblemas tende a crescer consideravelmente e buscar qual seria o melhor agrupamento de forma exaustiva, analisando todas as possibilidades, se torna algo inviável. Propõe-se, portanto, utilizar um algoritmo genético para definir o melhor agrupamento de nós da árvore de cenários em sub-árvores que serão resolvidos como subproblemas. O algoritmo otimiza a variável individuo, previamente apresentada, buscando o menor tempo de solução do problema. Como existe a necessidade de se resolver o problema de planejamento para um mesmo sistema várias vezes, espera-se que baste uma única execução do algoritmo genético para se definir o agrupamento ótimo para o sistema. Este agrupamento ótimo poderá então, ser repetido para as demais resoluções deste sistema, garantindo assim a melhor distribuição de subproblemas e um melhor tempo computacional. Na próxima seção o algoritmo implementado para alcançar este objetivo será apresentado.

\subsection{Técnicas inteligentes}

De acordo com a teoria algorítmica [GANDOMI, 2012], um problema torna-se difícil quando o aumento do tamanho do problema causa um aumento exponencial na complexidade do tempo. Portanto, surgiram vários métodos que tentam resolver aproximadamente esses problemas, esses métodos procuram soluções de maneira inteligente, ou seja, heurística, ao invés da busca exaustiva na qual avaliaria-se todas as soluções.

Ao longo da história, os mecanismos heurísticos mais poderosos foram inspirados pela natureza. Alguns exemplos são os Algoritmos 


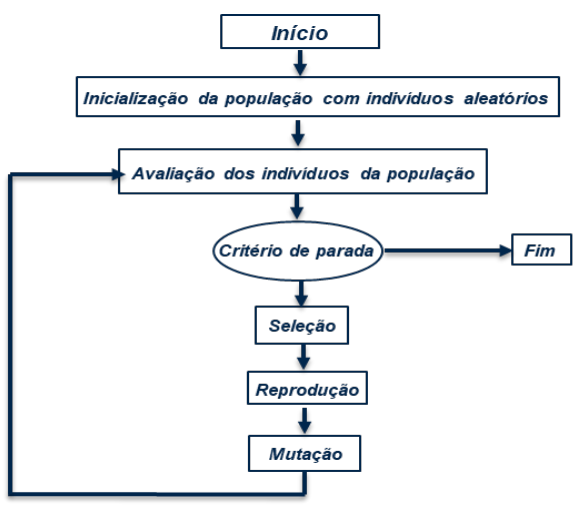

Fig. 6. Fluxograma Algoritmo Genético

Evolucionários (AE) [EIBEN, 1998] que imitam a teoria darwiniana da evolução, e Inteligência de Enxame que encontra inspiração dentro de uma teoria coletiva, descentralizada e auto-organizada [BLUM, 2011]. Embora atualmente existam muitas variantes diferentes de AE's, a ideia comum por trás de todas essas técnicas permanece a mesma: dentro da população de indivíduos, as soluções, a pressão ambiente provoca a seleção natural, sobrevivência do indivíduo, causando um aumento na tensão do população [EIBEN, 2003]. Os AE's determinam a qualidade das soluções candidatas, podendo ser o valor mínimo ou máximo da função objetiva relacionada à um problema a ser resolvido. Para o trabalho, a técnica escolhida foi á de algoritmo genético (GA) devido à natureza binária da variável a ser otimizada, variavel individuo apresentada no item anterior. A figura 6 apresenta o fluxograma do algoritmo genético implementado.

No algoritmo implementado, o usuário define o tamanho da poupulação, número de indivíduos que ela terá e a quantidade de gerações que serão analisadas. Com estas informações, o algoritmo constroi populações de índivíduos por meio de sorteio e os avaliam através da solução do problema de planejamento energético do sistema teste pela metodologia PDD paralelizada de forma assíncrona para o agrupamento de nós representado por aquele índíviduo, como mostrado no exemplo da figura 4. O algoritmo busca o menor tempo de solução, portanto os índivíduos que possuem menor tempo são os mais bem avaliados na etapa de avaliação e terão maior probabilidade de serem selecionados na etapa da seleção, etapa que compara os índivíduos da população e seleciona os de melhor desempenho para passarem pela etapa de reprodução, responsável pela criação dos índivíduos da nova população. Para evitar que a solução do problema fique presa em ótimos locais, a etapa de mutação adiciona índivíduos de forma aleatória à nova população. Todas essas etapas são repetidas com a nova população criada até que o critério de parada seja atingido. Os critérios de paradas definidos para o algoritmo foram o número total de gerações definidos pelo usuário e um critério de estabilidade, caso o valor ótimo global se repita durante cinco gerações consecutivas.

A próxima seção irá abordar os resultados das metodologias implementadas no trabalho. As convergências das metodologias PDD, implementada de forma recursiva, e PDD paralela assíncrona, a eficácia do algoritmo genético em encontrar o melhor agrupamento de subproblemas, e os ganhos computacionais da metodologia PDD paralela assíncrona.

\section{RESULTADOS}

\subsection{Sistema Teste}

A fim de validar a metodologia proposta e comparar os gastos computacionais da mesma, definiu-se um problema teste de instância mínima, uma usina hidrelétrica e duas termelétricas. O usuário determina o número de estágios e de aberturas a serem consideradas para a montagem da árvore de cenários, além de qual usina hidrelétrica do sistema brasileiro deseja utilizar no problema.
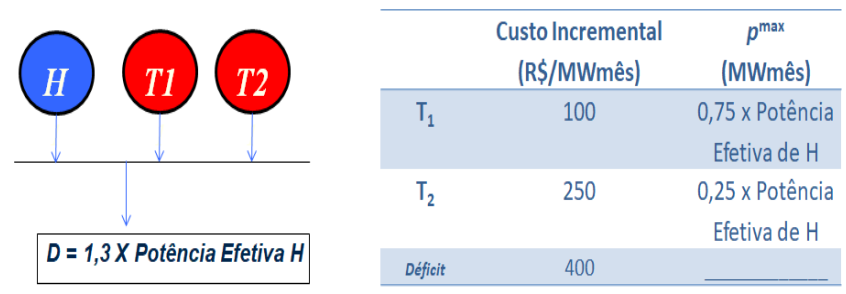

Fig. 7. Sistema teste

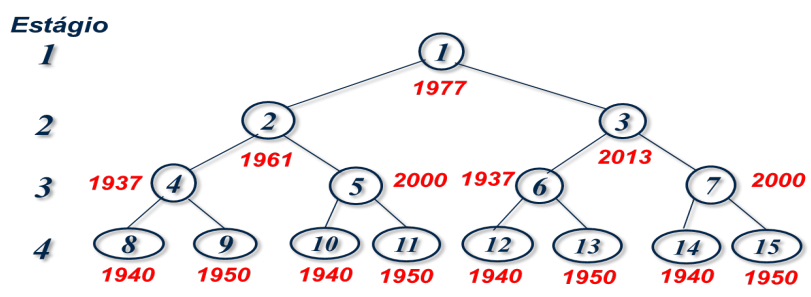

Fig. 8. Árvore de cenários para 4 estágios e 2 aberturas

A partir dos dados da usina hidrelétrica escolhida pelo usuário e lidos pelo algoritmo diretamente dos arquivos do Programa Mensal de Operação Energética (PMO) disponível em [PMO, 2019], são definidos os dados das usinas termelétricas e de demanda do sistema. Para os testes realizados, as potências máximas das térmicas foram estabelecidas como sendo setenta e cinco por cento e vinte e cinco por cento da potência efetiva da hidrelétrica escolhida, além disso a demanda do sistema é definida como sendo cento e trinta por cento a potência efetiva da usina hidrelétrica. A figura 7 representa o sistema teste juntamente com os dados de custos incrementais das térmicas e custo de déficit considerados para o mesmo.

Para construir a árvore de cenários a serem resolvidos utilizou-se o histórico de vazões encontrados nos arquivos do PMO, um sorteio entre os dados históricos foi realizado para definir as afluências à usina hidrelétrica em cada nó da árvore, como mostra a figura 8. Observa-se que as afluências das aberturas de cada estágio são iguais, para o caso da figura 8, como são apenas duas aberturas são sorteados apenas dois valores de afluência para cada estágio.

As restrições consideradas para o problema foram as restrições de atendimento a demanda, de balanço hídrico e de canalização das variáveis de decisão do problema. A formulação base do problema a ser resolvido pode ser visto nas equações 3 e 4 .

$$
\min \sum_{j} \sum_{i}\left(c_{g t 1} \times g t_{1 j}^{i}+c_{g t 2} \times g t_{2 j}^{i}+c_{d e f} \times d e f_{j}^{i}\right)
$$

sujeito a: $g t_{1 j}^{i}+g t_{2 j}^{i}+\rho \times v_{t j}^{i}+d e f_{j}^{i}=D_{j}^{i}$

$$
\begin{aligned}
v_{f j}^{i}+v_{t j}^{i}+v_{v j}^{i}-V I_{j}^{i} & =A F L_{j}^{i} \\
& x_{\operatorname{minj}}^{i} \leq x_{j}^{i} \leq x_{\operatorname{maxj}}^{i}
\end{aligned}
$$

onde: $\mathrm{i}=$ número do estágio; $\mathrm{j}=$ número da abertura $\mathrm{gt}_{1 j}^{i}=$ variáveis de decisão da geração da térmica 1 $g t_{2 j}^{i}=$ variáveis de decisão da geração da térmica 2 $v_{t j}^{i}=$ variáveis de decisão do volume turbinado da hidrelétrica $v_{f j}^{i}=$ variáveis de decisão do volume final da hidrelétrica $v_{v j}^{i}=$ variáveis de decisão do volume vertido da hidrelétrica $V I_{j}^{i}=$ valor do volume inicial da hidrelétrica $\rho=$ valor da produtibilidade da hidrelétrica $A f l_{j}^{i}=$ valor da afluência à usina hidrelétrica $d e f_{j}^{i}=$ variáveis de decisão do déficit

$D_{j}^{i}=$ valor da demanda do sistema 


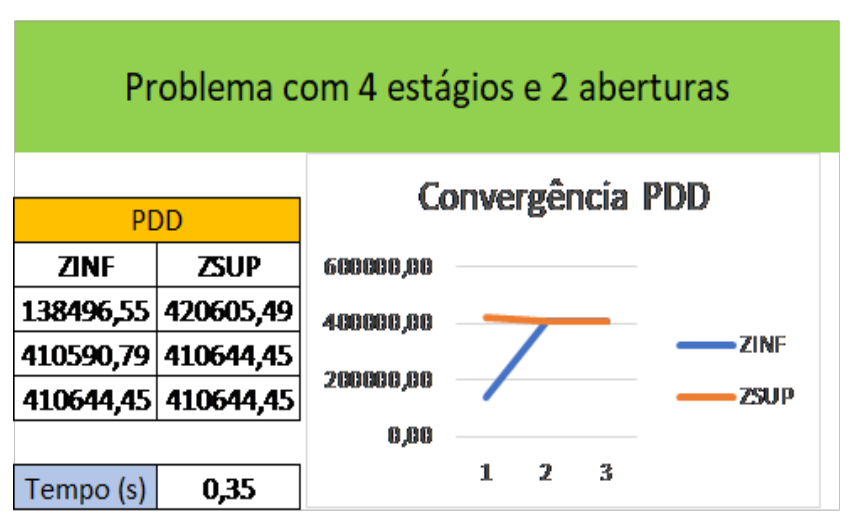

Fig. 9. Convergência PDD

\section{Problema com 4 estágios e 2 aberturas}

\begin{tabular}{|c|c|}
\hline \multicolumn{2}{|c|}{ PDD } \\
\hline ZINF & ZUP \\
\hline 210626,90 & 410644,53 \\
\hline 410644,45 & 410644,45 \\
\hline Tempo (s) & 0,21 \\
\hline Individuo & {$\left[\begin{array}{llll}1 & 1 & 0 & 1\end{array}\right]$} \\
\hline
\end{tabular}

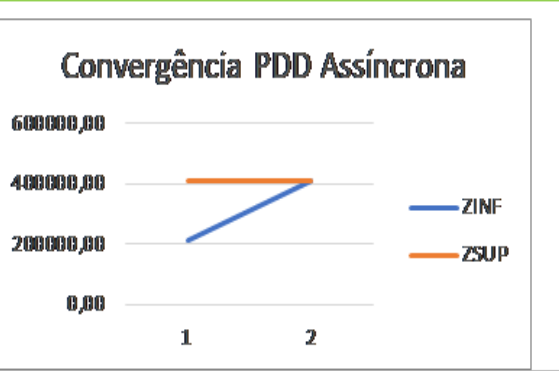

Fig. 10. Convergência PDD Assíncrona

\subsection{Convergência das Metodologias}

A Programação Dinâmica Dual (PDD) implementada apresentou convergência como esperado, a figura 9 apresenta a solução encontrada para um problema exemplo com quatro estágios e duas aberturas para a usina de Furnas.

A metodologia PDD paralela assíncrona também encontrou convergência para o mesmo problema apresentado anteriormente, como pode ser visto na figura 10. Além de alcançar a convergência, ela gastou uma iteração a menos do que a metodologia PDD tradicional e já obteve um tempo de solução menor do que a metodologia anterior apresentando um ganho computacional de 39,99\%.

\subsection{Algoritmo Genético}

Para testar a acurácia do algoritmo genético implementado, o problema de quatro estágios e duas aberturas foi resolvido 500 vezes para a mesma configuração, porém com valores de afluências à usina hidrelétrica diferentes. A figura 11 mostra os valores de tempo de solução encontrados para cada metodologia, PL, PDD e PDD paralela assíncrona. Todas as possibilidades de agrupamento de nós em sub-árvores foram consideradas para a resolução da PDD paralela assíncrona, os seus respectivos tempos estão discretizados de acordo com o individuo correspondente, figura 11.

Ainda na figura 11, em destaque vermelho temos o indivíduo encontrado pelo algoritmo genético como sendo o ótimo para cada execução, em 93,8\% dos casos o agrupamento encontrado pelo algoritmo foi o de menor tempo na rodada, validando assim sua utilização para definir o agrupamento de subproblemas a ser utilizado pela metodologia PDD paralela assíncrona.

\subsection{Ganho Computacional}

Para verificar a eficácia da metodologia proposta, o mesmo problema de instância mínima foi resolvido para árvores de cenários

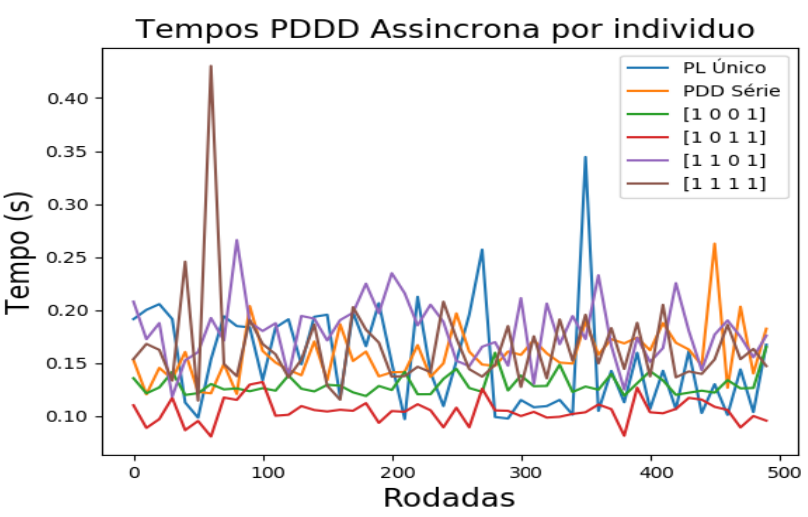

Fig. 11. Comparação dos tempos encontrados durante a solução do problema

\section{Problema com 6 estágios $\mathrm{e} 3$ aberturas}

\begin{tabular}{|c|c|}
\hline Metodologia & Tempo $(\mathrm{s})$ \\
\hline PL único & $\mathbf{0 , 1 9}$ \\
\hline PDD & $\mathbf{0 , 2 8}$ \\
\hline PDD Assíncrona & $\mathbf{0 , 1 5}$ \\
\hline \multicolumn{2}{|c|}{} \\
\hline Ganho Computacional (\%) \\
\hline PDD Assíncrona x PL & PDD Assíncrona x PDD \\
\hline $\mathbf{2 1 , 5 6 \%}$ & $\mathbf{4 7 , 5 5 \%}$ \\
\hline & \\
\hline Melhor Individuo & {$[\mathbf{1 1 1 0 0 1 ]}$} \\
\hline
\end{tabular}

\section{Comparaçäo ternpos de soluçäo}
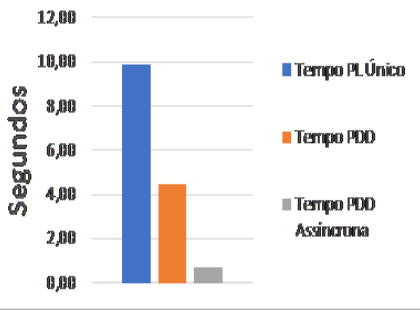

Fig. 12. Comparação dos tempos de execução para problema com 6 estágios

\section{Problema com 8 estágios e 2 aberturas}

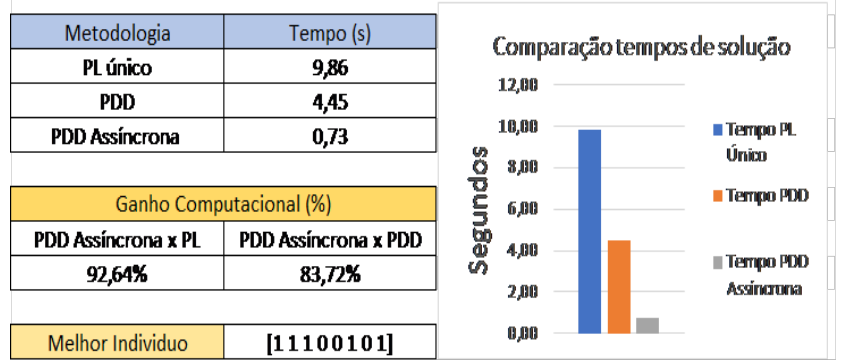

Fig. 13. Comparação dos tempos de execução para problema com 8 estágios

com mais estágios e aberturas, o que torna o problema bem mais complexo e aumenta consideravelmente o tempo para a convergência das metodologias tradicionais. Foi resolvido um problema com seis estágios e três aberturas, o que resulta na construção de 850 subproblemas e um problema com oito estágios e duas aberturas, o que resulta na construção de 509 subproblemas.

A figura 12 apresenta os resultados dos tempos gastos para a solução de um problema com seis estágios e três aberturas e a figura 13 para um problema com oito estágios e duas aberturas, ambos considerando a Usina Hidrelétrica Estreito. As figuras destacam também qual o agrupamento de nós foi considerado pela metodologia PDD assíncrona, explicitada pela variável individuo, para escolhê-lo foi utilizado o algoritmo genético como mostrado na seção 2.5. 
A metodologia implementada apresentou um ganho computacional de $47,55 \%$ quando comparado com a PDD tradicional para o problema com seis estágios e três aberturas e um ganho de $83,72 \%$ para o problema com oito estágios e duas aberturas, o que demonstra que quanto maior a complexidade do problema maior tende ser o ganho computacional da metodologia implementada.

\section{CONCLUSÕES E TRABALHOS FUTUROS}

Devido ao acoplamento temporal do planejamento da operação do Sistema Elétrico Brasileiro, as decisões tomadas hoje impactam diretamente nos custos operativos de um estágio futuro. Então, é de fundamental importância que as ações com relação às gerações hidrotérmicas sejam tomadas visando um período futuro, para que se tenha o mínimo custo operativo que atenda a demanda do mercado. Contudo, a definição das quantidades de geração das usinas hidrelétricas e termelétricas não é simples de ser encontrada, uma vez que as afluências às hidrelétricas futuras são desconhecidas e estas exercem influência direta sobre a geração hidrelétrica, cujo custo de operação é o mais barato.

Para a solução deste problema várias metodologias podem ser aplicadas, entre elas a Programação Dinâmica Dual (PDD), porém para problemas grandes, como o problema do sistema real brasileiro, esta metodologia pode demandar um esforço computacional elevado. O trabalho apresentou uma metodologia com o objetivo de obter ganhos computacionais ao ser comparada com as metodologias tradicionais. A metodologia consiste em realizar a paralelização assíncrona da PDD juntamente com o agrupamento inteligente, por meio de algoritmo genético, dos nós da árvore de cenários do problema em sub-árvores para serem distribuidas e serem resolvidas como subproblemas por diferentes processadores.

Os resultados mostram que a metodologia é eficaz para encontrar o ponto ótimo de operação dos sistemas e ao compará-la com as metodologias tradicionais podemos verificar que ela apresenta significante redução no tempo computacional necessário para atingir convergência do problema, principalmente à medida que a árvore de cenários se torna mais complexa. Como trabalho futuro, propõe-se um aprimoramento no algoritmo proposto de forma que o mesmo seja capaz de explorar todas as possíveis decomposições da árvore de cenários do problema, decomposição por nó, decomposição por cenário, por sub-árvore, e por cenário completo, como apresentado em [JESÚS, 2008]. Além disso, propõe-se a utilização da metodologia para a resolução de problemas mais complexos, de forma a aproximar o problema analisado ao planejamento energético do sistema brasileiro.

\section{AGRADECIMENTOS}

Este trabalho teve o suporte financeiro da Coordenação de Aperfeiçoamento de Pessoal de Nível Superior (CAPES), do Conselho Nacional de Desenvolvimento Científico e Tecnológico (CNPq), do Centro de Pesquisas de Energia Elétrica (CEPEL), da Fundação de Amparo à Pesquisa do Estado de Minas Gerais (FAPEMIG), do Instituto Nacional de Energia Elétrica (INERGE) e da Agência Nacional de Energia Elétrica (ANEEL).

\section{REFERÊNCIAS BIBLIOGRÁFICAS}

ONS, 2019. Operador Nacional do Sistema Elétrico. Web page: http://www.ons.org.br/paginas/sobre-o-sin/o-que-e-o-sin . Acessado: 2019-01-02.

MARCATO, A. L. M., (2002). Representação híbrida de sistemas equivalentes e individualizados para o planejamento da operação a médio prazo de sistemas de potência de grande porte. Rio de Janeiro: Tese de Doutorado, DEE, PUC-Rio.

M.E.P. Maceira, L.A. Terry, F.S. Costa, J. M. Damazio, A C. G. Melo, "Chain of optimization models for setting the energy dispatch and spot price in the Brazilian system", Proceedings of the Power System Computation Conference - PSCC'02, Sevilla, Spain, June 2002.

BENDERS, J. F., 1962, Partitioning procedures for solving mixedvariables programming problems", Numerische Mathematik, v. 4, n. 1, pp. 238252. ISSN: 0945-3245. doi: 10.1007/BF01386316.

J.R. Birge, "Decomposition and partitioning methods for multistage stochastic linear programs", Operations Research, v.33, n.5, pp. 9891007, 1985.

T. N. Santos, A. L. Diniz, C. T. Borges, "A New Nested Benders Decomposition Strategy for Parallel Processing Applied to the Hydrothermal Scheduling Problem", IEEE Transactions on Smart Grid, v. 8, n.3, pp. 1504-1512, 2017.

BRANDÃO, L.C. ; DINIZ, A.L. ; SIMONETTI, L. G. . Accelerating Dual Dynamic Programming for Stochastic Hydrothermal Coordination Problems. In: 20th Power Systems Computation Conference, 2018. Proceedings of the 20th Power Systems Computation Conference, 2018.

DEMPSTER, M.A.H; THOMPSON, R.T. EVPI-based importance sampling solution procedures for multistage stochastic linear programmes on parallel MIMD architectures. In: 1999 Annals of Operaions Research.

JESÚS M. Latorre, 2008, Analysis of stochastic problem decomposition algorithms in computational grids. Doi: 10.1007/s10479-0080476-1

DEMPSTER, M. A. H., THOMPSON, R. T., 1998, and aggregation of nested Benders decomposition", Annals of Operations Research, v. 81, n. 0, pp. 163188. ISSN: 1572-9338. doi: 10.1023/A:1018996821817. Disponível em: <http://dx.doi.org/10.1023/A:1018996821817>

A. H. Gandomi, X.-S. Yang, A. H. Alavi, and S. Talatahari. Bat algorithm for constrained optimization tasks. Neural Computing and Applications, pages 117, 2012, Article in press. Smith, S. e Jones, J. (2002). A paper on operations research. Pesquisa Operacional, $32: 5-44$.

A. E. Eiben and C. Schippers. On evolutionary exploration and exploitation. Fundamenta Informaticae, 35(1):3550, 1998.

C. Blum, J. Puchinger, G. Raidl, and A. Roli. Hybrid metaheuristics in combinatorial optimization: A survey. Applied Soft Computing, 11(6):41354151, 2011.

A. Eiben and J. Smith. Introduction to Evolutionary Computing. Springer-Verlag, Berlin, 2003.

PMO, 2019. PROGRAMA MENSAL DA OPERAÇÃO. Web page: http://ons.org.br. Acessado: 2019-01-02. 